\title{
An imaging spectrometer employing tunable hyperchromatic microlenses
}

\author{
Phuong-Ha Cu-Nguyen ${ }^{1}$, Adrian Grewe ${ }^{2}$, Patrik Feßer ${ }^{2}$, Andreas Seifert ${ }^{1}$, Stefan Sinzinger ${ }^{2}$ and Hans Zappe ${ }^{1}$ \\ We present the design, fabrication and characterization of hydraulically-tunable hyperchromatic lenses for two-dimensional (2D) \\ spectrally-resolved spectral imaging. These hyperchromatic lenses, consisting of a positive diffractive lens and a tunable concave \\ lens, are designed to have a large longitudinal chromatic dispersion and thus axially separate the images of different wavelengths \\ from each other. 2D objects of different wavelengths can consequently be imaged using the tunability of the lens system. Two \\ hyperchromatic lens concepts are demonstrated and their spectral characteristics as well as their functionality in spectral imaging \\ applications are shown.
}

Light: Science \& Applications (2016) 5, e16058; doi:10.1038/lsa.2016.58; published online 8 April 2016

Keywords: hyperchromatic lens; imaging spectrometer; tunable filter; tunable lens

\section{INTRODUCTION}

Chromatic aberration of a refractive lens is characterized by a wavelength-dependent focal length and is typically an undesirable phenomenon. As a result, numerous types of hybrid lenses, frequently consisting of two or more refractive or diffractive components, have been conceived to reduce or eliminate this effect ${ }^{1,2}$. However, some applications can take advantage of the chromatic aberration, using dispersion to realize micro-spectrometers or micro spectral filters. For example, a diffractive micro-Fresnel lens with high dispersion has been used with a movable pinhole along the axial axis to filter out the intensity of a certain wavelength, producing a highly compact and cost-efficient spectrometer ${ }^{3,4}$. In chromatic confocal imaging or surface profile sensing, the variably focused wavelengths are encoded for depth measurement. By using a diffractive optical zone plate or a combination of refractive elements with large chromatic aberration, three-dimensional imaging without the axial scanning as required in conventional confocal microscopes can be accomplished ${ }^{5-7}$. Finally, large chromatic aberration has also been used for quantitative phase imaging 8,9 .

Waller et al. ${ }^{8}$ have developed a new method for high-resolution quantitative phase imaging by utilizing the chromatic dispersion of a fixed borosilicate crown glass (BK-7, Schott AG, Mainz, Germany) refractive lens and a standard color camera in a $4 f$ system configuration. Using an approach employed by techniques such as phase contrast electron microscopy, multiple images at various values of defocus are captured to determine how the intensity of the wave varies along the optical axis, from which the phase information of the incident wave can be derived. By taking advantage of the chromatic aberration of the lens, different defocused and focused images are captured without physical movement. The phase information can be computed using the modified transport of intensity equation.
Increasing the chromatic dispersion can provide better signal-tonoise ratio in the derivative measurement ${ }^{8}$.

Another approach for quantitative phase microscopy was introduced by Harm et al. ${ }^{9}$ by using a hybrid diffractive-refractive optical lens doublet with tunable chromatic dispersion. The lens system consists of a varifocal Moiré Fresnel diffractive lens and a tunable liquid lens. The working principle is also based on separating the focused and defocused images, from which the phase objects can be numerically calculated. The focal length of both lenses can be changed, thus allowing variation of the chromatic dispersion while maintaining the constant focal length for the center wavelength. This approach helps the lens adapt to different samples where the phase gradient varies and the chromatic dispersion needs to be sufficiently large to allow numerical post-processing.

In the concept presented here, instead of capturing the focused and defocused images in one frame, we aim to utilize the high chromatic dispersion of a lens to axially separate and discretely collect only the focused images at different narrow wavelength bands. When combining all of those narrow-band images into a hyperspectral datacube, the spectrum for each pixel of the image can be extracted as has been shown in previous work ${ }^{10-12}$. The lens we employ to achieve this functionality, namely a tunable hyperchromatic lens (HCL), has a changeable focal length and a chromatic dispersion designed to be as large as possible. We first discuss the theoretical background concerning the relation between the chromatic dispersion and the resolved spectral resolution and subsequently present two fully-integrated designs of the tunable HCL including an outline of the required fabrication processes. The two lenses are both spectrally characterized and the results show a good functionality in confocal spectral filtering as well as in two-dimensional (2D) spectral imaging.

${ }^{1}$ Gisela and Erwin Sick Chair of Micro-optics, Department of Microsystems Engineering, University of Freiburg, Freiburg 79110 , Germany and ${ }^{2}$ Fachgebiet Technische Optik, Institut fur Mikro- und Nanotechnologien, Technische Universitat IImenau, IImenau 98684, Germany

Correspondence: H Zappe, Email: zappe@imtek.uni-freiburg.de

Received 17 June 2015; revised 23 November 2015; accepted 4 December 2015; accepted article preview online 5 December 2015 


\section{MATERIALS AND METHODS}

\section{Hyperchromatic lens concept}

The hyperchromatic lens, on which we base the spectral imaging system, is a combination of a diffractive lens and a hydraulicallytunable refractive lens. The tunable lens consists of a liquid-filled microfluidic cavity bounded by a distensible polymer membrane, which forms the refractive surface. By changing the pressure on the liquid in the cavity, the curvature of the membrane changes, allowing concave and convex profiles with a tunable radius of curvature and thus focal length ${ }^{13,14}$.

The diffractive part is designed as a Fresnel lens with a focal length strongly dependent on wavelength. Due to the high dispersion, the



Figure 1 Schematic diagrams of the two hyperchromatic lens concepts: (a) the 'discrete HCL' with separate hydraulically-actuated refractive and fixed

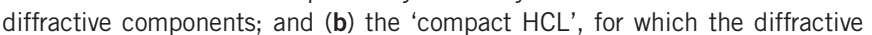
structure is etched onto the flexible refractive membrane. The curvatures of the refractive lenses are deformed by changing the pressure on an optical liquid in the lens chamber, thus tuning the focal length of hyperchromatic lenses. diffractive lens is employed to focus different wavelengths at different positions distributed along the optical axis. When the chromatic dispersion is broad enough, a certain wavelength can be focused to a point on an image plane while other wavelengths are strongly defocused, yielding a very low intensity for these at any given position on the plane.

The HCL itself is then a combination of these refractive and diffractive components. The refractive lens, operated with a variable concave profile, is joined to the diffractive component and the system focal length is tuned using hydraulic actuation. Due to the high chromatic aberration, only a narrow wavelength band is focused on the optical axis for a given focal length, and the wavelength at a given point is then tuned as the system focal length is tuned. With this configuration, thus, no physical translation of the lens components is needed for the wavelength scanning.

We develop here two designs for the hyperchromatic lens, namely a discrete HCL and a compact HCL, as illustrated in Figure 1. The discrete HCL is based on a static Fresnel diffractive lens fabricated on quartz glass and the tunable membrane-based liquid lens. In contrast, the compact HCL is fabricated by integrating the structure of the Fresnel lens onto the polymer membrane of the tunable liquid lens itself, hence providing a highly-compact HCL.

The working principle of a tunable HCL for chromatic confocal sensing is shown in Figure $2 \mathrm{a}$ and that for a hyperspectral imaging system is shown in Figure 2b. In the confocal approach, a HCL is used to disperse and focus the light from a white light point source on the optical axis. By placing a confocal pinhole in the image plane of the lens, a narrow spectral band can be filtered out as shown in Figure 2a. A detector behind the pinhole is used to measure the intensity of this wavelength. By tuning the refractive lens and scanning through different wavelengths, the complete spectrum of the incident light can be reconstructed ${ }^{3}$. As shown on the right of Figure $2 \mathrm{a}$, the narrow

a

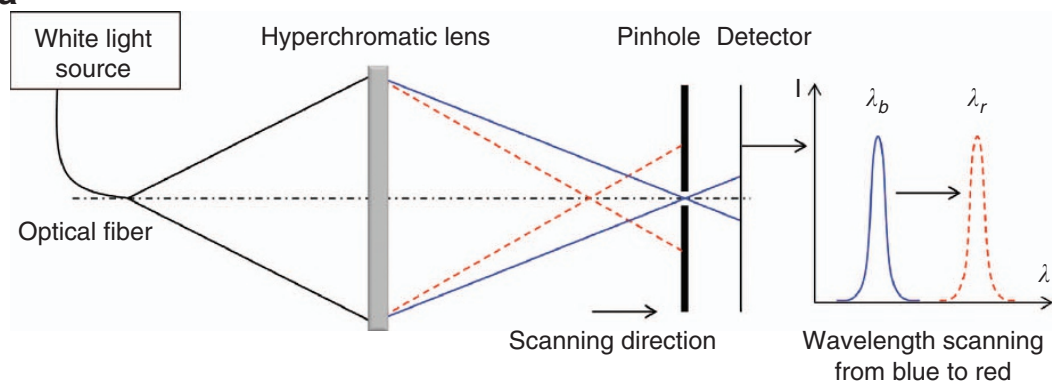

b

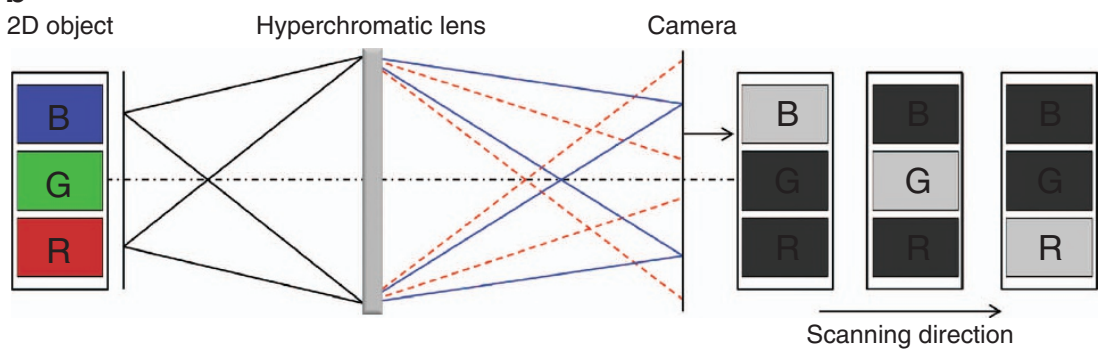

Figure 2 Working principles of a tunable hyperchromatic lens used for: (a) chromatic confocal sensing and (b) hyperspectral imaging. In a, a pinhole is placed in the image plane for spatial spectral filtering while the tunable lens is used for the wavelength scanning. Before tuning: the blue wavelength is focused on the pinhole and appears as a narrow spectral band on the detector; after tuning: the red wavelength is in focus on the pinhole so the spectral signal moves to the red wavelength. In $\mathbf{b}$ an object including red/green/blue parts is imaged by a hyperchromatic lens. The blue part is in focus while others are strongly blurred due to the high chromaticity; by tuning the lens, the green and red parts sequentially appear in focus on the camera. 
a Hyperchromatic lens

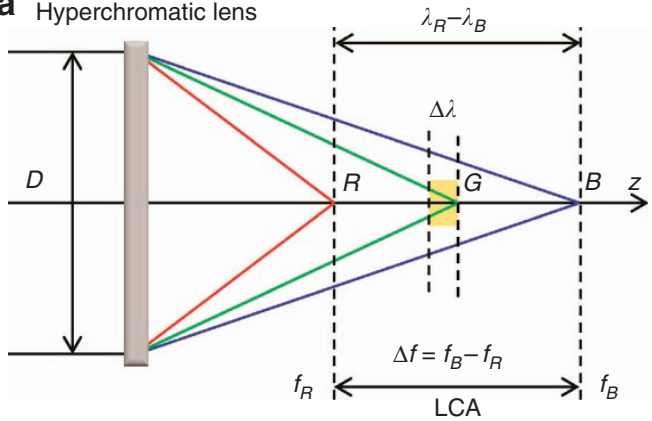

b

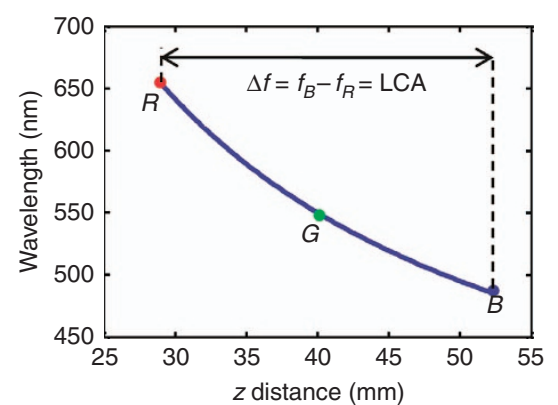

C

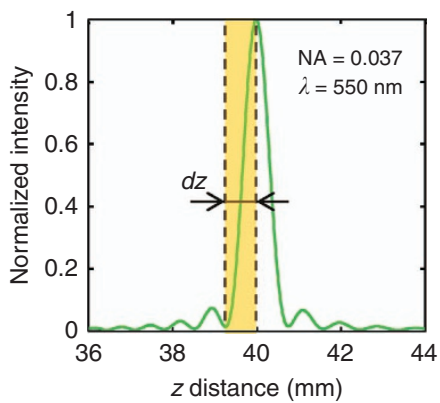

Figure 3 (a) Dispersion of the hyperchromatic lens with red/green/blue wavelengths focused on the optical axis $z$, when the lens is positioned at $z=0$; (b) distribution of the different wavelengths on the $z$ axis; and (c) the intensity distribution of a single wavelength on the $z$ axis where $d z$ is the distance between the peak maximum and the first minimum of the curve.

spectral band on the detector moves from blue to red wavelengths when tuning the focal length of the HCL.

The hyperspectral imaging for $2 \mathrm{D}$ object can be realized with the configuration shown in Figure $2 \mathrm{~b}$. When imaging an object with the HCL, only a single wavelength is in focus on the camera at any time. The out-of-focus wavelengths also contribute light to the image, but the intensity of the focused wavelength strongly dominates the intensity of the out-of-focus wavelengths. Therefore, the HCL can generate the sharp image of an object in a narrow spectral band even without using a pinhole for spectral filtering. By scanning through the spectral range, the images in narrow bands can be obtained and the spectra for each pixel on the image can be derived. The spectral resolution of the HCL will be discussed in the next section.

\section{Theoretical considerations}

Chromatic aberration. The Fresnel diffractive lens in the HCL system is configured as a circular structure with a focal length $f_{\text {diff }}$ at wavelength $\lambda$ given by

$$
\frac{1}{f_{\text {diff }}(\lambda)}=\frac{\lambda}{f_{0} \lambda_{0}}
$$

where $f_{0}$ is the designed focal length at the design wavelength $\lambda_{0}$.

On the other hand, the focal length $f_{\text {ref }}$ of the tunable refractive lens is given by the Lensmaker's formula

$$
\frac{1}{f_{\text {ref }}(\lambda)}=(n(\lambda)-1)\left(\frac{1}{R_{1}}-\frac{1}{R_{2}}\right)
$$

where $n(\lambda)$ is the refractive index of the liquid inside the lens (typically water) at wavelength $\lambda$, and $R_{1}$ and $R_{2}$ are the curvatures of the front and back surfaces of the lens, respectively.

Assuming that the diffractive and refractive lenses are thin lenses and in close contact, the effective focal length $f_{\text {eff }}$ of the HCL is given, as a function of wavelength, as

$$
\begin{gathered}
\frac{1}{f_{\text {eff }}(\lambda)}=\frac{1}{f_{\text {diff }}(\lambda)}+\frac{1}{f_{\text {ref }}(\lambda)} \\
f_{\text {eff }}(\lambda)=\left(\frac{\lambda}{f_{0} \lambda_{0}}+(n(\lambda)-1)\left(\frac{1}{R_{1}}-\frac{1}{R_{2}}\right)\right)^{-1}
\end{gathered}
$$

When the HCL is illuminated by a polychromatic plane wave, the focal points of different wavelengths are distributed on the optical axis, as shown in Figure 3a, and the relationship between the focal length and the wavelength is shown in Figure $3 \mathrm{~b}$. We see that the $\lambda-z$ correlation is nearly linear, with a correlation coefficient of $R^{2}=0.99$.
The longitudinal chromatic aberration (LCA) of the lens is defined as the difference between the focal lengths at the red and blue Fraunhofer lines (656.2 and $486.1 \mathrm{~nm}$, respectively), that is, LCA= $f=f_{B}-f_{R}$. A high value for LCA is an important property of the hyperchromatic lens since it acts as the dispersive element in the spectral imager. The LCA of a lens system was derived by Kingslake and Johnson ${ }^{15}$ and can be rewritten for the HCL as

$$
f=f_{\text {eff }}^{2}\left(\frac{1}{f_{\text {diff }} V_{\text {diff }}}+\frac{1}{f_{\text {ref }} V_{\text {ref }}}\right)
$$

where $V_{\text {diff }}$ and $V_{\text {ref }}$ are the Abbe numbers of the diffractive and refractive lenses, respectively.

The Abbe number of a refractive lens $V_{\text {ref }}$ in the visible wavelength range is given by

$$
V_{\mathrm{ref}}=\frac{n_{e}-1}{n_{F}-n_{C}}
$$

where $n_{F}, n_{e}$ and $n_{C}$ are the refractive indices of the lens material at the Fraunhofer F-, e- and C-lines, respectively.

In contrast, the Abbe number of a diffractive lens is given by

$$
V_{\mathrm{diff}}=\frac{\lambda_{e}}{\lambda_{F}-\lambda_{C}}
$$

where $\lambda_{F}, \lambda_{e}$ and $\lambda_{C}$ are again defined as above.

In this work we used a positive diffractive lens with a negative Abbe number $\left(V_{\text {diff }}=-3.452\right)$ and a negative (concave) refractive waterfilled lens with a positive Abbe number $\left(V_{\text {ref }}=58\right)$; both values are defined for the visible wavelength range. The combination of those lenses will yield a high absolute value of LCA as is required for this application.

For the simplified case, where the dispersion of the refractive part of the lens can be neglected, combining Equations (1) and (7) then allows us to write the LCA for the HCL as

$$
f=f_{\text {eff }}^{2}\left(\frac{1}{f_{\text {diff }} V_{\text {diff }}}\right)=f_{\text {eff }}^{2}\left(\frac{\lambda_{F}-\lambda_{C}}{f_{0} \lambda_{0}}\right)
$$

Spectral resolution. To determine the spectral resolution of the HCL, we consider the case where it is illuminated by a monochromatic plane wave. The intensity distribution for a single wavelength on 
the optical $z$ axis for an ideal lens with the paraxial approximation was derived by $\mathrm{Webb}^{16}$ and the axial resolution was given by

$$
d z=\frac{2 \lambda}{\mathrm{NA}^{2}}
$$

where NA is the numerical aperture of the lens. A calculated value is shown in Figure $3 \mathrm{c}$ for a wavelength of $550 \mathrm{~nm}$ and an NA of 0.037 ; the axial resolution $d z$ is found to be about $800 \mu \mathrm{m}$.

If we assume that two wavelengths are well resolved on the optical axis when the maximum of one coincides with the first minimum of the other (the typical Rayleigh criterion), thus the distance $d z$ between two peaks represents the limit of the spectral resolution ${ }^{3}$. When using the approximate linear correlation between wavelength and focal length as seen in Figure $3 \mathrm{~b}, d z$ can then be used to define the spectral resolution $\Delta \lambda$, given by

$$
\Delta \lambda=\frac{d z}{\Delta f}\left(\lambda_{R}-\lambda_{B}\right)
$$

Replacing Equations (8) and (9) into Equation (10), $\Delta \lambda$ can be rewritten as

$$
\Delta \lambda=\frac{2 \lambda}{a^{2}} f_{0} \lambda_{0}
$$

where $a$ is the radius of the lens.

We see thus that the main factors affecting the spectral resolution are the size of the lens system and the design parameters of the diffractive lens. Fabricating a large-diameter Fresnel lens can thus improve the spectral resolution but realizing a short-focal length diffractive lens with a large diameter, implying high numerical aperture, requires that small feature sizes be resolved over a large area, which is demanding on the fabrication technology. The minimum feature size that can easily be defined with the technologies available to us was $0.8 \mathrm{~m}$. In the following demonstrator, we therefore used a Fresnel lens with four phase levels, a diameter of $3 \mathrm{~mm}$, a focal length of $20 \mathrm{~mm}$ and a maximum numerical aperture of 0.17 . According to Equation (11), this lens is expected to yield a spectral resolution of $5.4 \mathrm{~nm}$ at the design wavelength of $550 \mathrm{~nm}$. Resolution will decrease as wavelength shifts from this design value.

We note that the spectral resolution derived above is based on the intensity distribution on the optical axis, that is, assuming an infinitely small detector. In experimental measurements, where the light is of course detected in a finite area, the spectral resolution is thus expected to be larger than the value calculated ${ }^{3}$.



\section{Lens design}

Refractive component. We now turn to the numerical design of the refractive and diffractive components of the HCL. As we saw above, the tunable refractive structure is a liquid membrane-based lens as shown schematically in the inset of Figure 4a. The magnetic actuator, which we will describe in the Fabrication and assembly section below, is employed to tune the lens by pumping the optical liquid into and out of the lens chamber. Design considerations for this refractive component have been extensively discussed elsewhere $e^{13,14}$ and the HCL structure uses this standard design.

However, detailed knowledge of the actual refractive membrane curvature is required for the design optimization of the diffractive component, considered in Diffractive component section below. Therefore, the variation of lens curvature as a function of hydraulic actuation level was measured using a stylus profilometer; the profiles are plotted in Figure 4b. An eighth-order polynomial function is used to fit the surface profile based on this measured data, and is given by

$$
h_{\mathrm{ref}}(\rho)=\sum_{i=1}^{8} \alpha_{i} \rho^{i}
$$

where $\rho$ is the radius of the lens aperture and the $\alpha_{i}$ are the polynomial fit coefficients, which are calculated and given in Table 1 . These values allow an accurate numerical representation of the refractive profile as a function of lens tuning and is required as input for the design optimization of the diffractive component, discussed in the following section.

Diffractive component. The profile of the Fresnel lens is typically described by its required phase function, which may be represented by a higher order polynomial, namely

$$
\varphi(\rho)=\sum_{i=1}^{N} A_{i} \rho^{2 i}
$$

where $\rho$ is the radius of the lens and $A_{i}$ are coefficients which can be freely set to optimize the imaging quality. In our case, the phase function was designed to yield the smallest focus spot size on the optical axis for an object at infinity. The phase function is then transformed into a surface relief profile, as shown in Figure $4 \mathrm{~b}$, which is given by

$$
h_{\text {diff }}(\rho)=\frac{1}{\left(n_{0}-1\right) 2 \pi}(\varphi(\rho) \bmod 2 \pi)
$$

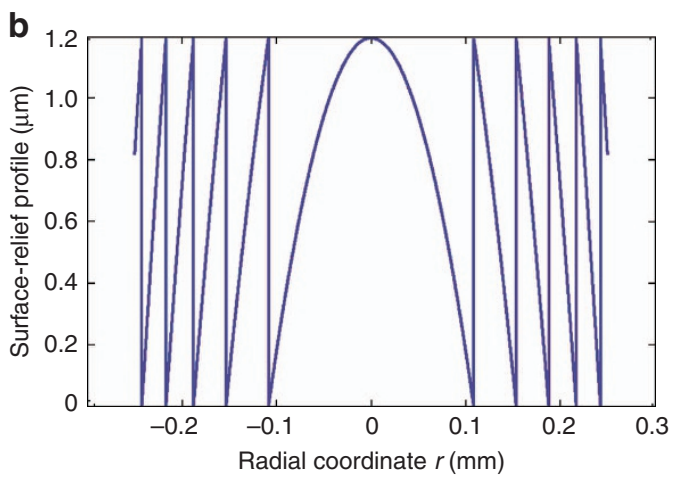

Figure 4 Lens profiles. (a) Measured concave refractive membrane profiles of the hydraulically tunable lens at different actuation settings, as measured by a stylus profilometer; the current values are those of the magnetic actuator; (b) the calculated profile of the diffractive Fresnel lens, calculated from the phase function. 
Table 1 Polynomial coefficients for modeling the refractive membrane curvatures of the hydraulically tunable lens; calculated by MATLAB as fit to the measured data from Figure $4 a$

\begin{tabular}{|c|c|c|c|c|c|c|c|c|c|}
\hline Current (mA) & $\alpha_{8}$ & $\alpha_{7}$ & $\alpha_{6}$ & $\alpha_{5}$ & $\alpha_{4}$ & $\alpha_{3}$ & $\alpha_{2}$ & $\alpha_{1}$ & $\alpha_{0}$ \\
\hline 0 & 7.3e-05 & $-5.6 e-05$ & $-2.4 \mathrm{e}-04$ & $1.6 e-04$ & $2.3 e-04$ & $-1.0 \mathrm{e}-04$ & $9.7 e-04$ & $8.6 e-05$ & $-2.6 e-03$ \\
\hline 22 & $2.6 e-05$ & $-1.9 e-05$ & $-6.8 \mathrm{e}-05$ & $2.9 e-05$ & $6.2 \mathrm{e}-05$ & $2.6 \mathrm{e}-05$ & $2.3 e-03$ & $-1.8 \mathrm{e}-04$ & $-5.6 e-03$ \\
\hline 45 & $3.4 \mathrm{e}-05$ & $2.9 \mathrm{e}-05$ & $-1.0 \mathrm{e}-04$ & $-1.0 \mathrm{e}-04$ & 1.0e-04 & $1.3 e-04$ & $7.4 \mathrm{e}-03$ & $8.8 \mathrm{e}-05$ & $-1.7 e-02$ \\
\hline 65 & $3.4 \mathrm{e}-06$ & $5.5 e-05$ & $1.4 \mathrm{e}-05$ & $-2.0 \mathrm{e}-04$ & $-1.9 \mathrm{e}-06$ & 2.0e-04 & $1.3 e-02$ & $-7.9 \mathrm{e}-06$ & $-3.0 e-02$ \\
\hline 86 & $-4.6 e-06$ & $5.6 e-05$ & $6.9 e-05$ & $-2.2 \mathrm{e}-04$ & $-1.0 \mathrm{e}-04$ & $2.1 \mathrm{e}-04$ & $1.8 \mathrm{e}-02$ & $2.2 \mathrm{e}-04$ & $-4.1 \mathrm{e}-02$ \\
\hline 102 & $-2.8 e-05$ & $3.4 \mathrm{e}-05$ & $1.6 e-04$ & $-1.5 e-04$ & $-1.9 e-04$ & $9.1 \mathrm{e}-05$ & $2.2 \mathrm{e}-02$ & 8.0e-04 & $-5.1 \mathrm{e}-02$ \\
\hline 117 & $-5.4 e-05$ & 7.1e-05 & $2.9 \mathrm{e}-04$ & $-2.8 \mathrm{e}-04$ & $-3.6 e-04$ & $1.7 \mathrm{e}-04$ & $2.6 \mathrm{e}-02$ & 1.0e-04 & $-6.0 \mathrm{e}-02$ \\
\hline 129 & $-2.1 e-04$ & $1.3 e-04$ & $8.8 \mathrm{e}-04$ & $-4.1 \mathrm{e}-04$ & $-1.1 \mathrm{e}-03$ & $-3.4 \mathrm{e}-05$ & 3.0e-02 & $-3.2 \mathrm{e}-04$ & $-6.7 e-02$ \\
\hline
\end{tabular}

The current values are those of the magnetic actuator.

Table 2 Comparison of the effective focal length, longitudinal chromatic aberration, axial resolution, and spectral resolution of the discrete and compact HCL structures

\begin{tabular}{lcccccc}
\hline & $\mathbf{D}(\mathrm{mm})$ & $\boldsymbol{f}_{\text {diff }}(\mathrm{mm})$ & $\boldsymbol{f}_{\text {ref }}(\mathrm{mm})$ & $\boldsymbol{f}_{\text {eff }}(\mathrm{mm})$ & $\Delta \boldsymbol{f}(\mathrm{mm})$ & $\boldsymbol{d z}(\mathrm{mm})$ \\
\hline Discrete HCL & 3 & 20 & -55 & 31.4 & 15 & 0.48 \\
Compact HCL & 3 & 20 & -55 & 31.5 & 15 & 5.4 \\
\hline
\end{tabular}

All values are given in unit $\mathrm{mm}$, except the spectral resolution.
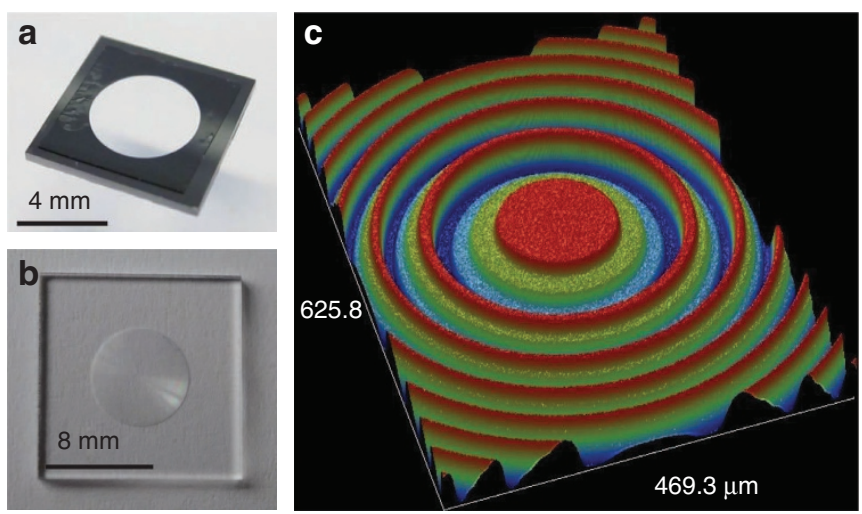

Figure 5 The diffractive and refractive components for the discrete $\mathrm{HCL}$ : (a) the Si aperture for the refractive lens with the circular aperture etched by DRIE and the polymeric membrane bonded on top; (b) the diffractive Fresnel lens fabricated on quartz glass; and (c) the center part of the Fresnel lens measured by white light interferometry.

where $n_{0}$ is the refractive index of the lens material at the design wavelength $\lambda_{0}$.

As mentioned above, the diffractive lens is optimized to obtain the smallest spot size of an object at infinity, which leads to a small value for axial resolution $d z$ and thus a high spectral resolution $\Delta \lambda$. The optimization process, performed using tracing-software (Zemax, Zemax, Kirkland, WA, USA), was done on the entire diffractive/ refractive system with a flat membrane (radius of curvature infinite) for the tunable refractive lens and a wavelength of $550 \mathrm{~nm}$. The root mean square value of the focal spot size on the optical axis was used as the merit function for the optimization. The variables in the procedure are the coefficients $A_{i}$ of the diffractive lens as given in Equation (13) with $N=3$, which then yield an optimized phase function

$$
\varphi(\rho)=-3826.597 \rho^{2}+30.979 \rho^{4}-0.480 \rho^{6}
$$

Incorporating Equation (15) into Equation (14) then yields the diffractive Fresnel height profile, optimized for the case, where the refractive lens has a focal length of infinity. Wave-optical simulations show that for non-infinite membrane curvatures of the refractive lens, given by fits to the measurement data (Table 1), the axial resolution $d z$ increases, but does not degrade appreciably compared to the theoretical optimum.

Hybrid HCL. As described in the Hyperchromatic lens concept section, two HCL concepts were developed: the discrete HCL, in which the diffractive and refractive components are separate entities, as modeled above; and the compact HCL, for which the diffractive Fresnel structure was patterned onto the refractive membrane. We consider here a comparison of the spectral resolution at the wavelength of $550 \mathrm{~nm}$ of these two HCL modalities. We use the diffractive lens described in Equation (15) and the water lens (refractive index $n=1.33$ ) with the membrane curvature at the actuation current of $129 \mathrm{~mA}$ in Table 1 .

For the discrete HCL, we assume that the diffractive and refractive lenses are thin ideal lenses and in close contact, thus the equations for the ideal lens presented above are applicable and employed to identify the LCA and the distance $d z$. For the compact HCL, on the other hand, we employed a wave-optical simulation, as developed by Thiele et $a .^{17}$ and based on evaluation of the Huygens-Fresnel diffraction integral, to estimate the intensity distribution along the optical axis. Based on the intensity distribution of the three primary wavelengths (red/green/blue) on the optical axis, the LCA and the distance $d z$ are determined.

The design parameters and simulated performance results, including the effective focal length, longitudinal chromatic aberration, axial resolution and spectral resolution for the discrete and compact HCL, are shown in Table 2. We see that, with the same design parameters, the spectral resolution limits for the discrete and compact HCL structures almost identical, despite the fact that the diffractive structure is designed for a flat substrate. As the refractive lens in the compact HCL is tuned, the Fresnel lens is deformed, but we see that the degradation in performance is minimal since its simulated 
spectral resolution, $5.5 \mathrm{~nm}$, is very close to the value of $5.4 \mathrm{~nm}$ determined for the ideal lenses of the discrete HCL.

\section{Fabrication and assembly}

The fabrication of the diffractive lens and the silicon aperture for the refractive lens employ standard high-precision microfabrication techniques; photographs of these two elements are shown in Figure 5 for the discrete HCL. The rigid lens frame, on which the polymeric membrane is suspended, is fabricated by micro-machining with potassium hydroxide $(\mathrm{KOH})$ etching $400 \mu \mathrm{m}$ deep from the back side and deep reactiveion etching (DRIE) etching through from the front side to create the circular aperture $3 \mathrm{~mm}$ in diameter.

The diffractive lens is designed as a positive lens with the focal length of $20 \mathrm{~mm}$ at the wavelength of $550 \mathrm{~nm}$, and optimized for the diffraction limited on the optical axis with the phase function shown in Equation (15). The lens is fabricated on quartz glass by 2-step lithography using inductively coupled plasma (ICP)-reactive-ion etching (RIE) etching, thus forming a 4-level structure to enhance the diffraction efficiency; a detail of the Fresnel structure imaged using white light interferometry is shown in Figure $5 c$.

The design and assembly process for the discrete HCL with the integrated actuator are summarized in Figure 6a. The lens chamber and the actuation reservoir are generated by successively stacking different layers of a laser-patterned adhesive transfer tape (3M 467MP 200MP,
3M Center, St Paul, MN, USA) and a Poly(methyl methacrylate) (PMMA) baseplate together. The cavities are sealed by a polyacrylate membrane (3M VHB4905, 3M Center, St Paul, MN, USA) and the lens chamber is filled with deionized (DI) water. The quartz Fresnel lens is mounted on the PMMA base plate, outside the fluidic cavity.

As is indicated in Figure 6, the hydraulic lens tuned using a magnetic actuator, seen at the left of the schematic, consisting of a permanent $\mathrm{NeFeB}$ magnet mounted on a flexible membrane and a spiral coil configured outside the fluid chamber. By increasing or decreasing the current to the coil, the induced repulsive force on the magnet changes the pressure inside the fluid chamber, tuning the curvature of the refractive lens, at the right.

The fabrication and assembly process for the compact HCL are presented in Figure 6b. In this case, the lens is an all-silicone frame with the diffractive Fresnel structures replicated directly on the refractive membrane in a one-step molding process. The lens is designed to have a lens aperture of $10 \mathrm{~mm}$ and a focal length of $50 \mathrm{~mm}$ at the wavelength of $550 \mathrm{~nm}$. The metal mold for the replication is realized as shown in Figure $6 \mathrm{~b}$ with the diffractive optical elements quartz chip used as a master and aligned to the top of the refractive lens; a second planar glass plate is used to seal the hollow. The silicone elastomer polydimethylsiloxane (PDMS) Sylgard 184 is injected into the resulting mold and cured at 90 for $30 \mathrm{~min}$. The PDMS frame is subsequently removed from the mold and bonded onto a PMMA baseplate after being activated in
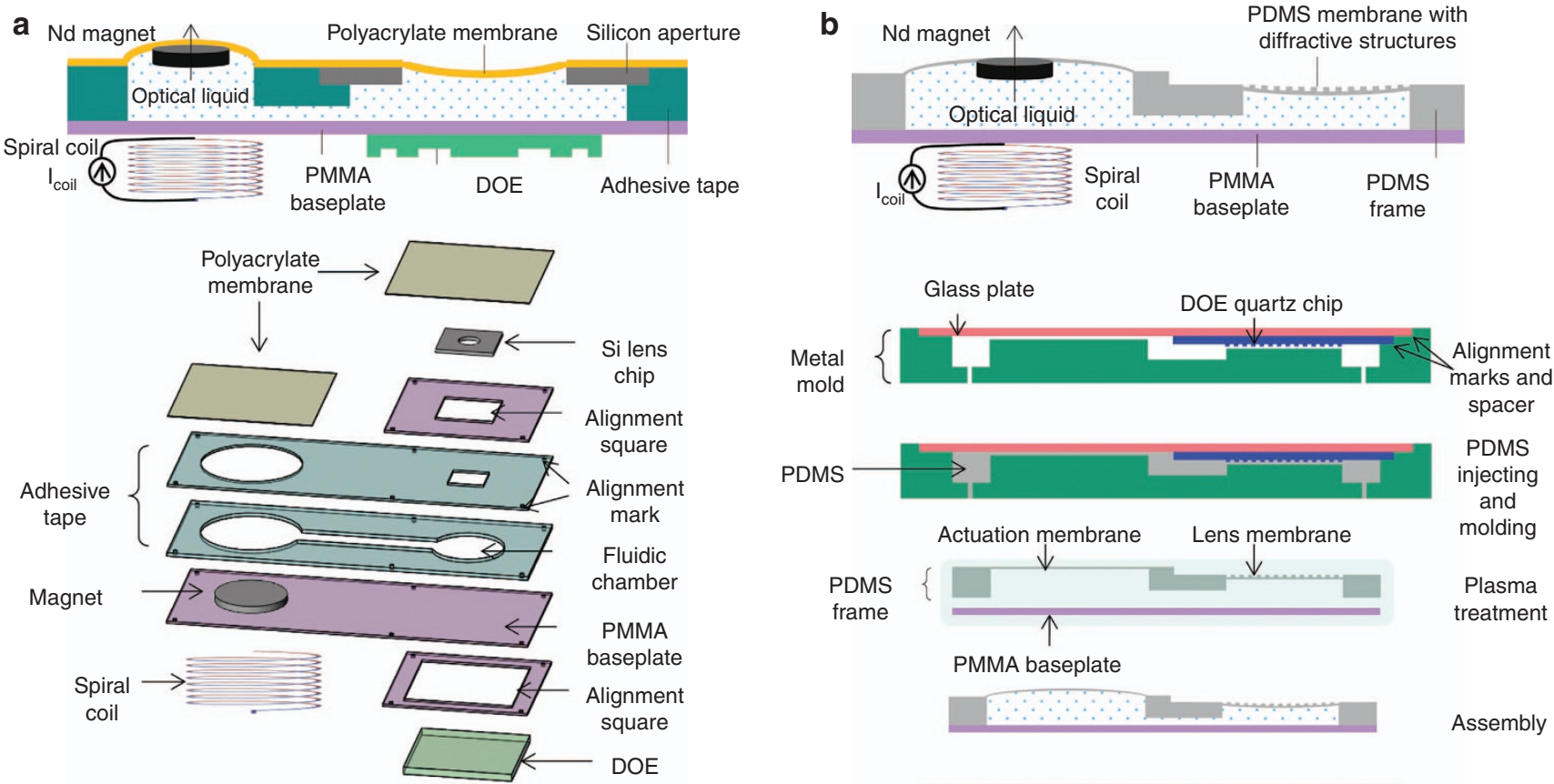

PMMA baseplate
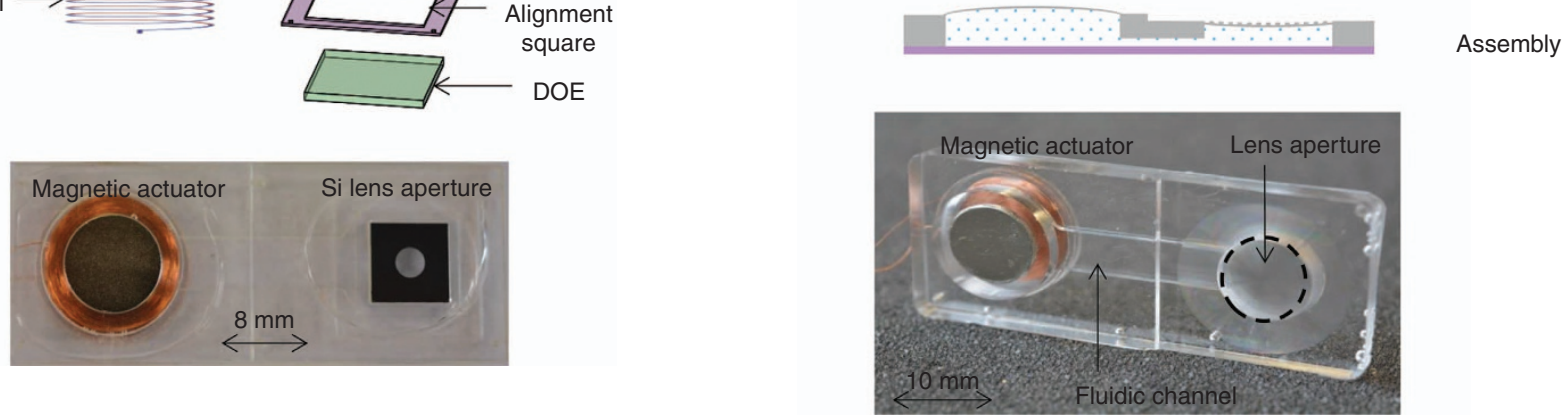

Discrete $\mathrm{HCL}$

Compact HCL

Figure 6 From top to bottom: schematic cross-sections; assembly processes; and photographs of the completed tunable hyperchromatic lenses with integrated magnetic actuators. From left to right: fabrication process for the discrete hyperchromatic lens (a) and fabrication process for the compact hyperchromatic lens (b). 
oxygen radio frequency plasma. The magnetic actuator is then integrated and the lens is filled with DI water using a needle and syringe.

Photographs of the discrete HCL and the compact HCL with fully integrated actuator are shown in the lower part of Figure 6.

\section{RESULTS AND DISCUSSION}

\section{Chromatic confocal measurement}

The optical configuration used to characterize the hyperchromatic lenses was seen in Figure 2a for both the discrete and compact HCL structures. The white light point source is the cleaved facet of a fiber, which is connected to a white light source with a broad emission spectrum and is confocally arranged with a pinhole in the image plane. The optical fiber and the detector pinhole both have a 50- $\mu \mathrm{m}$ diameter.

Based on the hyperchromatic properties of the lens, the white light is dispersed along the axial direction and the pinhole is used to spatially filter out a certain narrow wavelength range. Seen at the top of Figure 7 are the red/green/blue spots corresponding to the images of the detector pinhole at different actuation states of the lenses, we see clearly that different parts of the spectrum are transmitted. If the pinhole is replaced by an optical fiber facet connected to a spectrometer, the spectral bands shown in the lower part of Figure 7, taken for 10 discrete actuation states of the lens, result. The full width at half maximum of the individual signals, corresponding to the spectral resolution of the lens, are all between 12 and $14 \mathrm{~nm}$ for the discrete HCL and 10 and $18 \mathrm{~nm}$ for the compact HCL over the entire tuning range of 450-900 nm, showing the uniform response of the HCL over a wide spectral range.

These linewidth values are larger than those analytically predicted in the previous section. Reasons for this disparity include the fact that, experimentally, the signal is detected in an extended circular region (equal to the pinhole area), while in the theoretical analysis only the intensity exactly on the optical axis is considered.

For the performance comparison between two lens models, we see that during the tuning process, the spectral widths of the discrete HCL vary in a narrow range $(12-14 \mathrm{~nm})$, while that of the compact $\mathrm{HCL}$ fluctuate in wider range $(10-18 \mathrm{~nm})$. Moreover, from the spectral signal shown in the lower part of Figure 7, we see that the noise signal for the discrete HCL is less than that of the compact HCL. This can be visualized in the images of the white light point source in the upper part of Figure 7, where the discrete HCL gives sharper circular images when compared with those from the compact HCL. This difference can be explained by the fact that a possible slight misalignment between the fabricated diffractive structures and the lens aperture of the compact HCL has increased the stray light, and thereby broadened the transmitted bandwidth. Thus we believe that the performance of the compact HCL may be improved by use of a more precise fabrication process.

The actuation currents for tuning the transmission wavelength are given above the spectral peaks in Figure $7 \mathrm{a}$ and $7 \mathrm{~b}$. We notice that the current levels for the compact HCL are lower than for the discrete HCL, even though the same actuator concept is used. This difference is due to the fact that the PDMS membrane in the compact HCL is softer than the polyacrylate membrane used for the discrete HCL. The more pliable membrane leads to lower required currents, but may also yield an undefined curvature under some circumstances, thus leading to increased optical aberrations.

\section{D hyperspectral imaging application}

We demonstrate 2D hyperspectral imaging using a configuration similar to the optical layout shown in Figure 2b. The 2D object is an image on an LCD monitor, consisting of three different areas marked with different colors, namely red, green and blue, with RGB values of 255 on each channel. The objects and the reference spectra for each area are measured by the spectrometer and are shown in Figure 8a.

The object is then imaged by the hyperchromatic lenses. Due to the chromatic dispersion, one distinct narrow spectral band is focused on the camera at any given lens actuation level, forming a sharp image at that wavelength, while the other wavelengths are strongly defocused. As a result, the out-of-focus spectrum is not imaged and only a
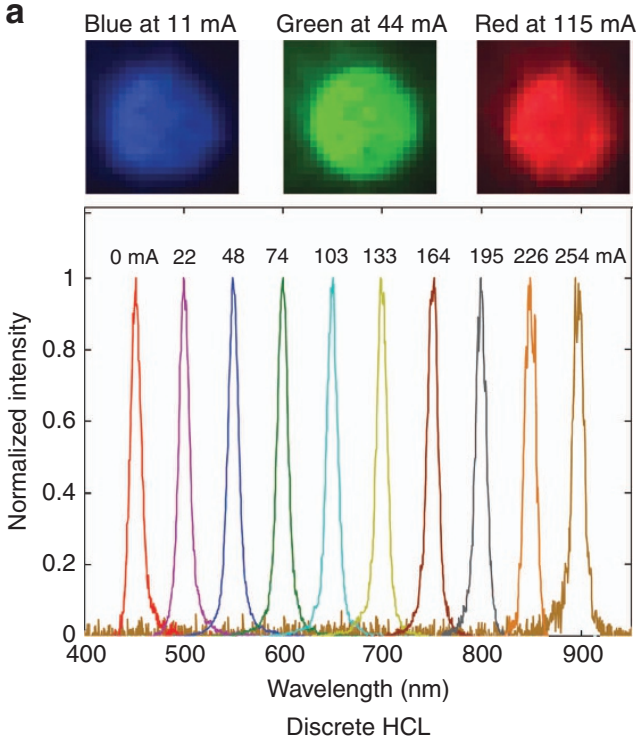

b
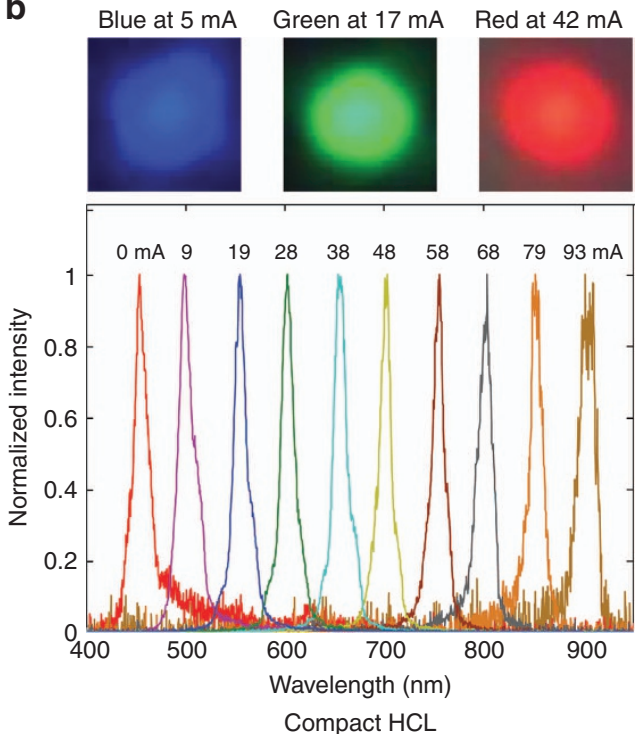

Figure 7 Chromatic confocal measurement results for the discrete $\mathrm{HCL}$ (a) and the compact HCL (b). The measurement setup is shown in Figure 2a where the white light point source is used. Upper parts are the images of the three colors (red, green and blue) dispersed from the white light source, focused on the pinhole and seen by a camera. The lower diagrams are the normalized spectral transmissions through the hyperchromatic lens at various actuating currents, detected by a spectrometer. 
a

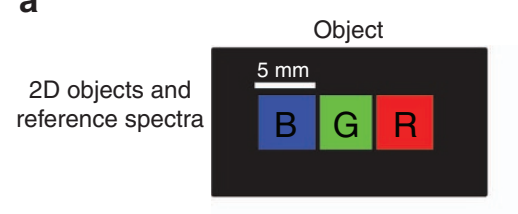

b

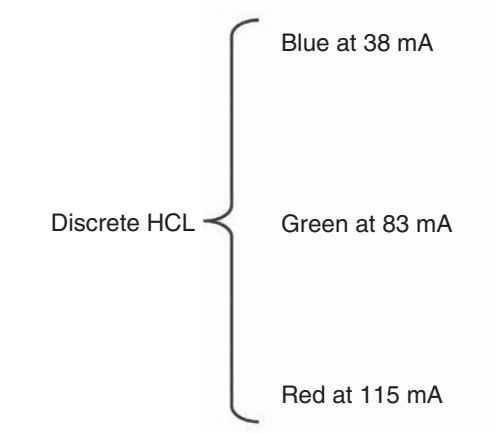

C

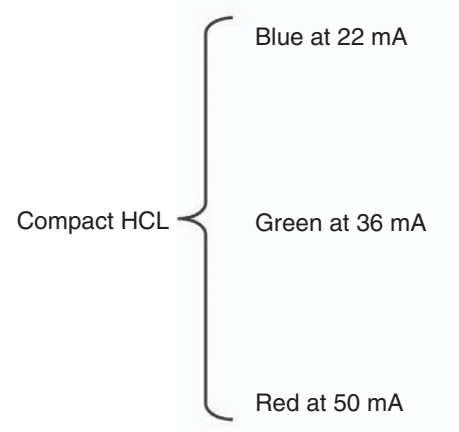

Images from normal lens
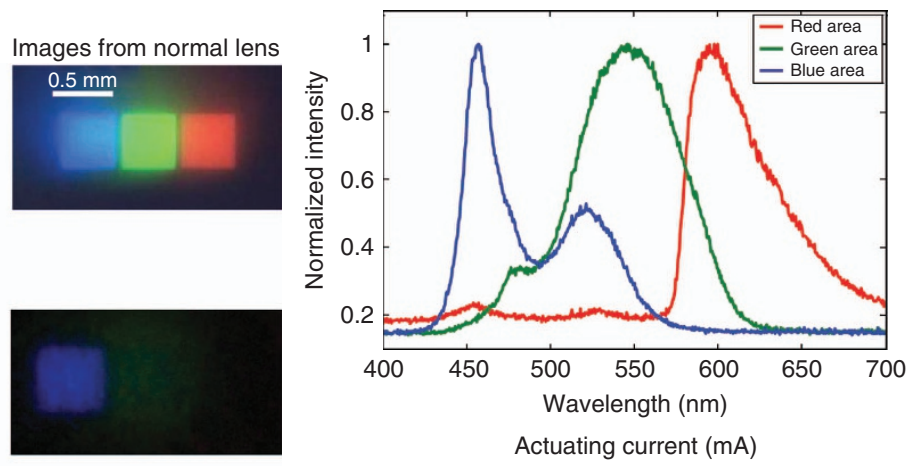

Actuating current (mA)
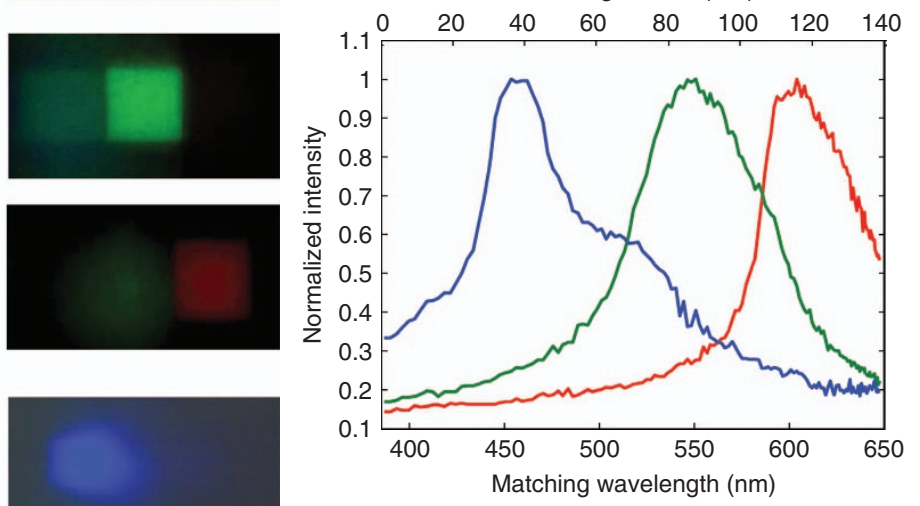

Actuating current (mA)



Figure 8 A two-dimensional (2D) hyperspectral measurement of an object consisting of three different colored areas. Reference spectra measured by a commercial spectrometer (a) and the hyperspectral images measured by the discrete HCL (b) and the compact HCL (c). The measurement setup is shown in Figure 2b. On the left: the objects imaged by the hyperchromatic lenses; seen here are three images for each lens at the best focus at different actuation currents; the magnification is 10:1. On the right: spectra of different areas on the object measured by the HCL; the intensity is measured in grayscale values as a function of the actuation current.

contributes to a low level of background noise. Tuning the HCL results in focusing a different image at other wavelengths on the camera image plane. Seen on the left are three images generated on the camera at different actuation states.

Each image delivers specific spectral information of the object, such that continuously tuning the HCL over the visible wavelength range yields a continuous range of spectral bands, from which the spectrum for each pixel in the spatial dimension can be composed. The spectra seen on the right of Figure $8 \mathrm{~b}$ and $8 \mathrm{c}$ show the intensity variations of three pixels in the middle of the red/green/blue areas when the HCL is tuned to those wavelengths. The intensities are measured in terms of gray scale values and are normalized with respect to the highest values; we see good correlation with the reference spectra measured by the commercial spectrometer and shown the top of the figure. The top axis shows the applied actuation currents and the bottom the corresponding wavelengths, indicating the nearly linear correlation between the actuation current and the wavelength as shown previously by $\mathrm{Cu}-\mathrm{Nguyen}$ et al. ${ }^{12}$.

When comparing the behavior of the discrete and compact HCL structures, we see that the $2 \mathrm{D}$ images generated by the compact $\mathrm{HCL}$ are blurred around the squares due to optical aberration resulting from the misalignment of the diffractive structures on the circular membrane as well as the deformation of those structures during the tuning process. In contrast, the images from the discrete HCL seem to be sharp, especially the image at the green wavelength, which is comparable to that generated by a normal glass lens as shown in Figure 2a.

Finally, an examination of the intensity profile across the square in the image shows that, if the magnification is unity, the HCL can resolve two adjacent squares with a spacing of $80 \mu \mathrm{m}$, where the intensity drop at the mid-point is $20 \%$, and the field of view can cover 
the entire $4.65 \times 3.72 \mathrm{~mm}$ image sensor. This spatial resolution can be improved by a more precise fabrication process.

\section{CONCLUSION}

In summary, the hyperchromatic lenses presented here enable continuous spectral filtering in a wide range of $450-900 \mathrm{~nm}$ with a spectral resolution of $13 \mathrm{~nm}$ in a confocal arrangement and a spatial resolution of $80 \mu \mathrm{m}$ in $2 \mathrm{D}$ imaging. These performance values compare favorably with other miniaturized MEMS-based spectrometer technologies, such as a hyper-spectral mosaic filter consisting of an array of discrete micro Fabry-Perot filters grouped into $4 \times 4$ pixels, yielding 16 bands between 465 and $630 \mathrm{~nm}$ with a spectral resolution of $11 \mathrm{~nm}$ and a spatial resolution limited by the size of filter group ${ }^{18}$. Tunable hyperchromatic lenses such as these are thus suitable for use in hyperspectral microscopy or as individual elements in a miniaturized hyperspectral sensor.

\section{CONFLICT OF INTEREST}

The authors declare no conflict of interest.

\section{ACKNOWLEDGEMENTS}

This work was funded by the German Federal Ministry of Education and Research. The authors thank Simon Thiele at the Laboratory of Optical Design and Simulation at the University of Stuttgart for the wave-optical simulation.

1 Zhou GY, Leung HM, Yu HB, Kumar AS, Chau FS. Liquid tunable diffractive/refractive hybrid lens. Opt Lett 2009; 34: 2793-2795.

2 Valley P, Savidis N, Schwiegerling J, Dodge MR, Peyman G et al. Adjustable hybrid diffractive/refractive achromatic lens. Opt Express 2011; 19: 7468-7479.

3 Kitaura N, Ogata S, Mori Y. Spectrometer employing a micro-Fresnel lens. Opt Eng 1995; 34: 584-588.
4 Park Y, Choi SH. Miniaturization of optical spectroscopes into Fresnel microspectrometers. J Nanophotonics 2013; 7: 077599.

5 Dobson SL, Sun PC, Fainman Y. Diffractive lenses for chromatic confocal imaging. Appl Opt 1997; 36: 4744-4748.

6 Papastathopoulos E, Körner K, Osten W. Chromatic confocal spectral interferometry. Appl Opt 2006; 45: 8244-8252.

7 Hillenbrand M, Mitschunas B, Brill F, Grewe A, Sinzinger S. Spectral characteristics of chromatic confocal imaging systems. Appl Opt 2014; 53: 7634-7642.

8 Waller L, Kou SS, Sheppard CJR, Barbastathis G. Phase from chromatic aberrations Opt Express 2010; 18: 22817-22825.

9 Harm W, Roider C, Jesacher A, Bernet S, Ritsch-Marte M. Dispersion tuning with a varifocal diffractive-refractive hybrid lens. Opt Express 2014; 22: 5260-5269.

10 Körner K, Kohler C, Papastathopoulos E, Ruprecht A, Wiesendanger T et al. Arrangement for rapid locally resolved flat surface spectroscopic analysis or imaging has flat raster array of pinholes turned about acute angle relative to spectral axis on detector matrix which fills up with elongated sumatrices. DE Patent App. DE 102006107172 A1, 2007.

11 Grewe A, Hillenbrand M, Sinzinger S. Bildgebende hyperspektrale Sensorik unter Einsatz verstimmbarer Optiken. Photonik 2013; 1: 38-41.

12 Cu-Nguyen PH, Grewe A, Hillenbrand M, Sinzinger S, Seifert A et al. Tunable hyperchromatic lens system for confocal hyperspectral sensing. Opt Express 2013; 21: 27611-27621.

13 Zhang W, Zappe H, Seifert A. Wafer-scale fabricated thermo-pneumatically tunable microlenses. Light Sci App/ 2014; 3: e145; doi:10.1038/lsa.2014.26.

14 Werber A, Zappe H. Tunable microfluidic microlenses. Appl Opt 2005; 44: 3238-3245.

15 Kingslake R, Johnson RB. Lens Design Fundamentals, 2nd edn. New York: Academic Press; 2010

16 Webb RH. Confocal optical microscopy. Rep Prog Phys 1996; 59: 427-471.

17 Thiele S, Seifert A, Herkommer AM. Wave-optical design of a combined refractivediffractive varifocal lens. Opt Express 2014; 22: 13343-13350.

18 Provoost J. Hyperspectral imager opens options for consumer, medical and industrial uses. Photon Spectra 2015; 49: 46-51.

(c) (1)(2) This work is licensed under a Creative Commons AttributionBY NC SA NonCommercial-ShareAlike 4.0 International License. The images or other third party material in this article are included in the article's Creative Commons license, unless indicated otherwise in the credit line; if the material is not included under the Creative Commons license, users will need to obtain permission from the license holder to reproduce the material. To view a copy of this license, visit http:// creativecommons.org/licenses/by-nc-sa/4.0/ 